\title{
Simulations of Galactic Cosmic Rays Impacts onto the Herschel/PACS Photoconductor Arrays with Geant4 Code
}

\author{
C. Bongardo (bongardo@pd.astro.it) \\ INAF-Osservatorio Astronomico di Padova, Vicolo dell'Osservatorio 5, I-35122 \\ Padova, Italy \\ P. Andreani (andreani@oats.inaf.it) \\ INAF-Osservatorio Astronomico di Trieste, via Tiepolo 11, I-34136 Trieste, Italy \\ Max-Planck fur Extraterrestrische Physik, Postfach 1312, D-85741 Garching, \\ Germany \\ G. De Zotti (dezotti@pd.astro.it) \\ INAF-Osservatorio Astronomico di Padova, Vicolo dell'Osservatorio 5, I-35122 \\ Padova, Italy
}

\begin{abstract}
We present results of simulations performed with the Geant4 software code of the effects of Galactic Cosmic Ray impacts on the photoconductor arrays of the PACS instrument. This instrument is part of the ESA-Herschel payload, which will be launched in late 2007 and will operate at the Lagrangian L2 point of the SunEarth system. Both the Satellite plus the cryostat (the shield) and the detector act as source of secondary events, affecting the detector performance. Secondary event rates originated within the detector and from the shield are of comparable intensity. The impacts deposit energy on each photoconductor pixel but do not affect the behaviour of nearby pixels. These latter are hit with a probability always lower than $7 \%$.

The energy deposited produces a spike which can be hundreds times larger than the noise. We then compare our simulations with proton irradiation tests carried out for one of the detector modules and follow the detector behaviour under 'real' conditions.
\end{abstract}

Keywords: Instrumentation: detectors, photoconductor - Galaxy: cosmic rays ISM: cosmic rays

\section{Introduction}

The European Space Agency Herschel satellite, scheduled for launch on August 2007 with an Ariane-5 rocket, will operate at the Lagrangian L2 point of the Sun-Earth system (see ESA web page: www.rssd.esa.int/SAgeneral/Projects/Herschel). Herschel is the fourth cornerstone mission of ESA Horizon 2000 program and will perform imaging photometry and spectroscopy in the far infrared and submillimetre part of the spectrum. The Herschel payload consists of two cameras/medium res-

(c) 2018 Kluwer Academic Publishers. Printed in the Netherlands. 
olution spectrometers (PACS and SPIRE) and a very high resolution heterodyne spectrometer (HIFI).

The PACS (Photo-conductor Array Camera and Spectrometer) instrument will perform efficient imaging and photometry in three wavelength bands within 60-300 $\mu \mathrm{m}$ and spectroscopy and spectroscopic mapping with spectral resolution between 1000 and 2000 over the entire wavelength range $(60-210 \mu \mathrm{m})$ or short segments.

PACS is made of four sets of detectors: two Ge:Ga photoconductor arrays for the spectrometer part and two Si-bolometer arrays for the photometer part. On each instrument side each detector covers roughly half of the PACS bandwidth (see Poglitsch et al., 2004). More about Herschel - PACS can be found in the PACS Web pages: pacs.mpegarching.mpg.de and pacs.ster.kuleuven.ac.be.

It is well known that cosmic rays may influence strongly the detector behaviour in space. The performances of detectors such as ISOCAM and ISOPHOT on board of the Infrared Space Observatory (ISO) were largely affected for time periods long enough to corrupt a large amount of data. Our goal, in the present paper, is to exploit our present knowledge on detectors and cosmic environment to understand how the detector performances change from the expectations.

In this paper we focus our attention on the GeGa photoconductor arrays (hereafter $\mathrm{PhC}$ ) of the PACS instrument, following its response under simulated cosmic rays irradiation. The detectors are similar to those on board of ISO and we expect therefore that they will be strongly affected by cosmic ray hitting.

In addition we compare proton irradiation tests, performed on a single module of the $\mathrm{PhC}$, with Geant4 simulations and draw conclusions about their performances.

The paper is organized as follows: in Sections 2 through 5, the used simulation toolkit, the input detector design, the Galactic Cosmic Ray spectra and the Physics List are outlined. In Section 6 we report the results. Section 7 deals with the irradiation tests which were performed at UCL-CRC on one detector module and the comparison with our simulations. In the final Section 8 we summarize the results reported in this draft.

\section{Geant4 Monte Carlo code}

Monte Carlo simulations were accomplished with the Geant $4^{1}$ (hereafter G4) toolkit, which simulates the passage of particles through

\footnotetext{
${ }^{1}$ Every simulation was run with the 7.0 patch 01 version, with CLHEP 1.8.1.0 with a gcc 3.2 .3 compiler
} 
matter. G4 provides a complete set of tools for all the domains of detector simulation: Geometry, Tracking, Detector Response, Run, Event and Track management, Visualization and User Interface. An abundant set of Physics Processes handle the diverse interactions of particles with matter across a wide energy range, as required by the G4 multidisciplinary nature; for many physical processes a choice of different models is available. For any further information see the Geant4 Home page at: geant4.web.cern.ch/geant4/.

\section{The Detector Design}

\subsection{PACS AS A SPECTROMETER}

The PACS employs two PhC arrays (stressed/unstressed) to perform imaging line spectroscopy and imaging photometry in the $60-300 \mu \mathrm{m}$ wavelength band. In spectroscopy mode, it will image a field of about $50 \times 50$ arcsec, resolved into $5 \times 5$ pixels, with an instantaneous spectral coverage of $\approx 1500 \mathrm{~km} / \mathrm{s}$ and a spectral resolution of $\approx 175 \mathrm{~km} / \mathrm{s}$ with an expected sensitivity ( $5 \sigma$ in $1 \mathrm{~h}$ ) of $3 \mathrm{mJy}$ or $2.5 \times 10^{-18} \mathrm{~W} / \mathrm{m}^{2}$.

The high stressed module is cooled down to $1.8 \mathrm{~K}$ (whereas the low stressed is cooled to $2.5 \mathrm{~K}$ ), the Front End Electronic (FEE) to $4 \mathrm{~K}$. All main metal components are made of a light metal alloy called Erg-Al $(\mathrm{AlZn} 5,5 \mathrm{MgCu})$.

\subsection{The Geometry}

Figures 1 and 2 show the modeled detectors with Geant4.

The most important part of the $\mathrm{PhC}$ is the cavity. Inside the cavity there is the active part of the $\mathrm{PhC}$, the pixel. Each pixel is made of Ge:Ga (in the high stress configuration Ga doping is $10^{14} \mathrm{~cm}^{-3}$, that is an atom of $\mathrm{Ga}$ for about $10^{9}$ atoms of $\mathrm{Ge}$; this negligible $\mathrm{Ga}$ concentration is not further considered here). The pixel is a simple box of $1 \times 1 \times 1.5 \mathrm{~mm}$. Each pixel is sustained by two boxes ('cubes') made of $\mathrm{CuBe} 2$ (a $\mathrm{Cu}, \mathrm{Be}$ and $\mathrm{Pb}$ alloy). On the external parts of these two last boxes there are two different cylinders made of $\mathrm{CuBe} 2$ (pedestal 1 and 2). A steel cylinder (the $\mathrm{kugel}$ ) is placed on the right-hand side of pedestal 1. A thinner and smaller cylinder is located in the left-hand side. The sequence of a single pixel is: kugel, pedestal 1, cube, pixel, cube and pedestal 2 with its thinner cylinder. Between two sequences of pixels there is an $\mathrm{Al}_{2} \mathrm{O}_{3}$ (Aluminum oxide) insulator. The sequence of the 16 pixels starts with an insulator and ends with a kugel. The pixels have a single face that is not shadowed by the cavity, that is, clearly, the one facing the fore optics (see below). 
The fore optics (hereafter FO) is the most difficult part to be designed with G4. Actually it is constructed as a unique block made of Erg-Al, from which they subtracted a cone non orthogonal to the respective pixel. We designed each cone individually, making use of the boolean solids. All the FO should be gold coated with a $8.95 \mathrm{~g}$ gold mass. Simple calculations of the surface area of the FO leads to a coating thickness of $\approx 1.6 \mu \mathrm{m}$. We did not reproduce such a coating.

The FEE is made of two different volumes: a box and a trapezoid, both $0.5 \mathrm{~mm}$ thick. The Erg-Al components of the module have three different thicknesses. The small cubes sustaining the FO are $1.75 \mathrm{~mm}$ thick; the end of the horseshoe is $2.3 \mathrm{~mm}$ thick; the horseshoe is 4.1 mm thick.

Figure 1 shows the geometry of the PACS PhC (the single stressed and unstressed modules), as drawn in G4. Figure 2 shows the whole stressed $\mathrm{PhC}$ detector. This geometry is that used by the G4 software for the simulations.

For the Galactic Cosmic Rays simulations we considered all the components 'external' to the detector, i.e. the PACS box, the cryostat and the spacecraft and called the ensemble 'shield' and approximated it with an Aluminum sphere, with thickness of $11 \mathrm{~mm}$ and at a temperature of $80 \mathrm{~K}$, which is the nominal temperature of the passive cooled Herschel telescope. The inner radius is $20 \mathrm{~cm}$ and the outer $21.1 \mathrm{~cm}$.

For the irradiation tests the external environment was modelled as close as possible to the real one and it is described in details in $\S 7$.

The G4 software then requires the design of a 'world volume' containing all the detector part and the specification of the environment in which the detector is placed. We made the world volume a little bit larger than the shield, i.e. is a sphere with a radius $21.2 \mathrm{~cm}$.

\section{Galactic Cosmic Rays spectra}

Herschel will be injected in a orbit around L2. The L2 environment, as regards the Galactic Cosmic Ray (hereafter GCR) rates, has been measured by the WMAP satellite (Prantzos 2004). This environment is similar to that of the geostationary orbit and therefore the GCR spectra are known. In the limit of the distance Earth-L2 the GCRs flux gradient is negligible. Therefore we use GCR fluxes of a geostationary orbit as a reasonable approximation. In particular we use the Cosmic Ray Effects on Micro-Electronics (CREME) model set both for the solar-minimum (GCR maximum) and for the solar-maximum environment (Nymmik et al. 1992). This model uses numerical models of the ionizing radiation 

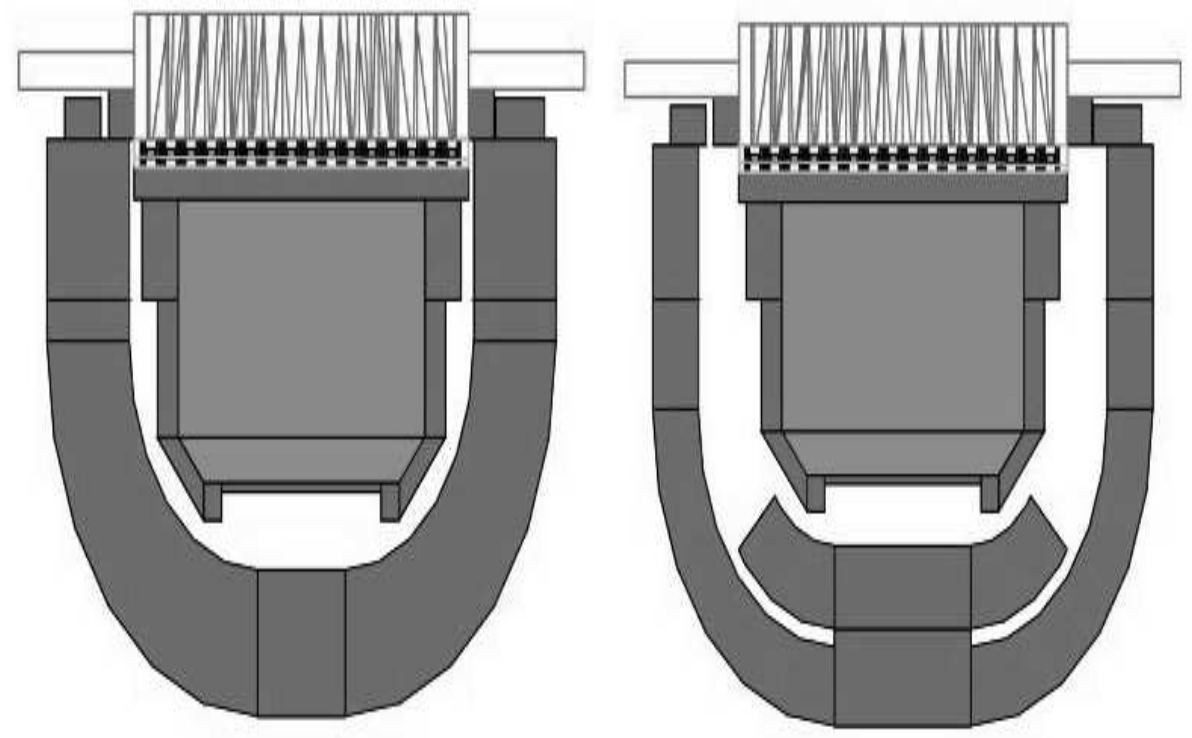

Figure 1. The PACS high (left) and low (right) stressed module (left).

Magenta: the fore-optics. Yellow: the gold coating of the FEE and the active pixel. Red: the $\mathrm{CuBe} 2$ contacts. Blue: the $\mathrm{Al}_{2} \mathrm{O}_{3}$ insulator. Gray: the cylindrical steel segment. Cyan: Erg-Al components.

environment in near-Earth orbits to evaluate the resulting radiation effects on electronic systems in space ${ }^{2}$.

A very diffuse background, called galactic vacuum by G4, which is a statistical view of the real interactions and diffusions of the Galactic Cosmic rays sources with and from the interstellar material, is defined and used as a good approximation of an empty space where an isotropic cosmic ray flux is present. This environment has the following physical properties:

$\mathrm{A}=1.01 \mathrm{~g} /$ mole (atomic mass)

$\mathrm{Z}=1$ (atomic number)

$\rho=1 \times 10^{-25} \mathrm{~g} / \mathrm{cm}^{3}$ (density)

$\mathrm{P}=1 \times 10^{-19} \mathrm{~Pa}$ (pressure)

Our simulation considers the most common GCRs in the near orbit Earth heliosphere: protons, alpha particles and nuclei of Li, C, N, O

${ }^{2}$ The CREME version used in this work is CREME94, which was more extensively tested and compared with measurements, however there is an updated version, CREME96, with a web page: https://creme96.nrl.navy.mil/ 


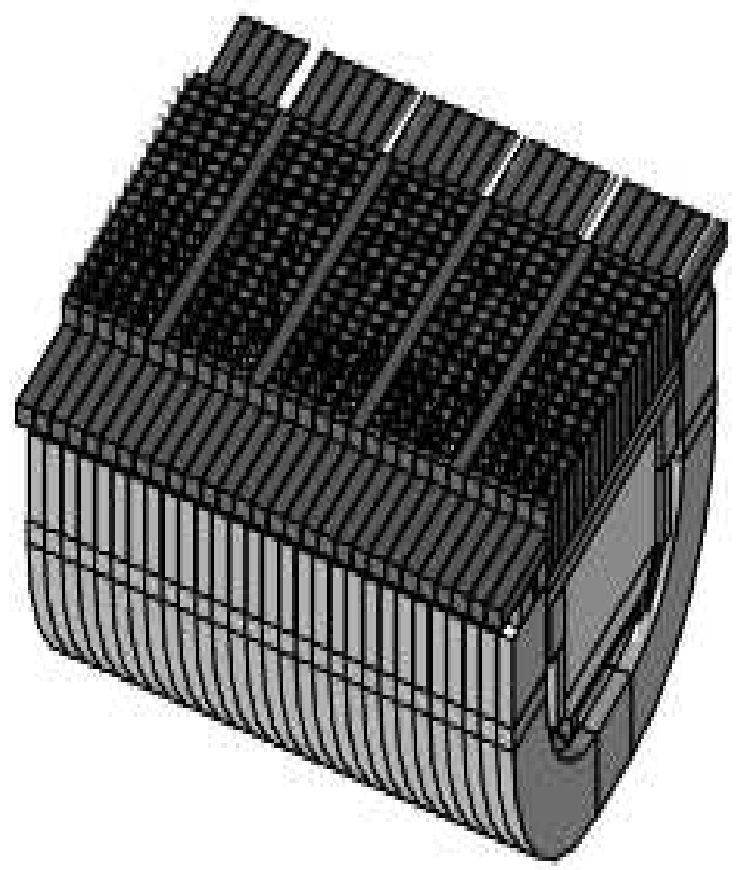

Figure 2. The PACS array. It contains 25 modules of 16 pixels each, making an array of $25 \times 16$.

(see Figure 3). It cannot include heavier nuclei, such as iron, since Fe physics has not yet been implemented in the Ion Physics of G4 (see $\S 5)$. In Table I we report the rates (number of particles per second) of each GCR type computed by integrating the fluxes reported in figure 3 over the shield surface and particle energy. Herschel will reach the L2 point in 2007 at a solar minimum but it will operate until an epoch of increasing solar activity. Therefore, we consider both solar activity phases.

\subsection{The Generate Source Particle Module}

G4 is built in such a way that once the geometry (volumes) and the physics are chosen and setup, the tool may be played from outside the main program through an User Interface (UI) easy accessible and modifiable (i.e. a macro file). In this macro file the user specifies: (a) the graphical visualization and/or (b) the type and the number of particle hitting the detector and their energy. One single particle with a determined energy represents an event. 
Table I. Rates (number of nuclei per second, on a sphere of $21.2 \mathrm{~cm}$ radius) for different GCR types and solar activity.

\begin{tabular}{lrr}
\hline & $\begin{array}{r}\text { Solar min } \\
\text { rates }(\# / s)\end{array}$ & $\begin{array}{r}\text { Solar max } \\
\text { rates }(\# / s)\end{array}$ \\
\hline $\mathrm{H}$ & 11275.89 & 4553.28 \\
$\mathrm{He}$ & 1102.56 & 458.67 \\
$\mathrm{Li}$ & 7.46 & 2.96 \\
$\mathrm{C}$ & 33.52 & 13.92 \\
$\mathrm{~N}$ & 8.96 & 3.68 \\
$\mathrm{O}$ & 31.31 & 13.00 \\
\hline
\end{tabular}


Figure 3. The differential GCR SEDs at the solar minimum (left) and maximum (right). Each curve corresponds to a type of particle (from top to bottom: proton, $\alpha, \mathrm{C}, \mathrm{O}, \mathrm{Li}, \mathrm{N})$. The rates reported in Table I are the integrals over energy and refer to the full shield surface.

We simulated the GCR seedings via the General Source Particle Module (GSPM; Ferguson 2000a,b,c), a code for the spacecraft radiation shielding, that is based on the radiation transport code G4. The user must specify the input parameters of the particle source: (1) the spatial and (2) the energy distribution. Every time G4 requires a new event, the GSPM randomly generates it according to the specified distributions. We adopt an angular distribution that is isotropic over a spherical surface (the vacuum sphere), without any preferred particle momentum. We then set the energy distribution of each particle according to the SEDs shown in Figure 3 (see also §5.2). 


\section{The Physics Model}

We had to define a list of physical interactions with the shielding and the different components of the photoconductors in order to best reproduce the impact of GCRs on the photoconductors. The list is comprehensive of all the possible physical interactions:

- Electro-magnetic physics: photo electric and Compton effect; pair production; electron and positron multiple scattering, ionization, bremsstrahlung and synchrotron; positron annihilation.

- General physics: decay processes.

- Hadron physics: neutron and proton elastic, fission, capture and inelastic processes; photon, electron and positron nuclear processes; $\pi^{+}, \pi^{-}, \mathrm{K}^{+}, \mathrm{K}^{-}$, proton, anti-proton, $\sigma^{+}, \sigma^{-}$, anti- $\sigma^{+}$, anti- $\sigma^{-}$, $\chi^{+}, \chi^{-}$, anti- $\chi^{+}$, anti- $\chi^{-}, \omega^{+}, \omega^{-}$, anti- $\omega^{+}$and anti- $\omega^{-}$multiple scattering and ionization. We added the High Precision Neutron dataset, that is valid for neutrons till $19.9 \mathrm{MeV}$.

- Ion physics: multiple scattering, elastic process, ionization and low energy inelastic processes. We added inelastic scattering (J.P. Wellish, private communication) using the binary light ion reaction model and the Shen cross section (Shen et al. 1989).

- Muon physics: $\mu^{+}, \mu^{-}$multiple scattering, ionization, bremsstrahlung and pair production; $\mu^{-}$capture at rest; $\tau^{+}$and $\tau^{-}$multiple scattering and ionization.

For 'ion physics' of alpha-particles, protons, and $\mathrm{H}$ isotope nuclei we used the cross section by Tripathi et al. (2000), holding for energies of up to $20 \mathrm{GeV}$ per nucleon. The cross sections used for the other ions (Shen et al. 1989) are valid only up to $10 \mathrm{GeV}$ per nucleon. This means that we had to cut out high energy ions of the GCRs (whose flux is lower than $10^{-5} / \mathrm{m}^{2} / \mathrm{s} / \mathrm{sr}$ ). The treatment of Ion Physics is strictly valid only for nuclei not heavier than $\mathrm{C}$. However we have applied it also to simulate $\mathrm{N}$ and $\mathrm{O}$, which should not behave much differently.

The model called by our physics list is different depending on the energy of the incoming particles. Our physics list is based on QGSP_BIC ${ }^{3}$ list (J.P. Wellish, private communication; see the Geant4 web page for details). Modifications to such a list are:

1. We added the High Precision Neutron dataset, which is valid for neutrons up to $19.9 \mathrm{MeV}$.

\footnotetext{
${ }^{3}$ Quark Gluon String Physics_Binary Cascade
} 
2. For neutrons and protons, we introduced ${ }^{4}$ the Bertini cascade (Bertini \& Guthrie 1979) up to $90 \mathrm{MeV}$ and made the BIC starting from 80 $\mathrm{MeV}$.

3. QGSP is assumed valid from 10 , rather than $12, \mathrm{GeV}$.

4. We have taken into account the gamma- and electro-nuclear reactions, so we considered the electromagnetic_GN physics list.

\subsection{ThrESHOLDS}

In order to avoid the infrared divergence, some electromagnetic processes require a threshold below which no secondary particles will be generated. Then, each particle has an associated production threshold, i.e. either a distance, or a range cut-off, which is converted to an energy for each material, which should be defined as an external requirement. A process can produce the secondaries down to the recommended threshold, and by interrogating the geometry, or by realizing when the mass-to-energy conversion can occur, recognize when particles below the threshold have to be produced.

We have set different production thresholds (hereafter cuts) for each geometrical region.

We decided: 1) not to use very small cut values (below 1-2 $\mu \mathrm{m}$ ), since this could affect the validity of physical models at such small steps (V. Ivantchenko, private communication); 2 ) to set cut values $\approx 1 / 5$ of the volume thickness. We identified groups of volumes with the same thicknesses: in particular we defined 6 different regions (see Table II). Results are robust since they do not depend on the cut values, both default and the chosen ones lead to similar outputs, with an increasing of the secondary event production. The results (energy deposition) among the different simulations were always comparable at $1 \sigma$. On the one hand this was expected, since most of the volumes have still the same default cut value, $0.7 \mathrm{~mm}$. On the other hand it is possible that Erg-Al thresholds have already achieved convergence at the default values, that is we are very slightly tuning them. Although results are pretty similar (see Table III) those with our chosen cuts allow a better tracking inside each detector volume.

\footnotetext{
${ }^{4}$ It is well known that (1) the BIC does not reproduce well data for energies below $50 \mathrm{MeV}$ (Ivanchenko et al. 2003). In contrast, the Bertini cascade works well below $50 \mathrm{MeV}$ for all but the lightest nuclei. And (2) the BIC for pions and kaons has not been tested yet (J.P. Wellish, private communication).
} 
Table II. The five regions of the photoconductor array and their specific cut values.

\begin{tabular}{lrl}
\hline Region & Cut Value & Thickness \\
\hline Shield & $2 \mathrm{~mm}$ & $11 \mathrm{~mm}$ \\
High_mm & $0.7 \mathrm{~mm}$ & $3.02-4.1 \mathrm{~mm}$ \\
Low_mm & $0.3 \mathrm{~mm}$ & $0.73-2.1 \mathrm{~mm}$ \\
High_mic & $100 \mu \mathrm{m}$ & $300-600 \mu \mathrm{m}$ \\
Low_mic_a & $5 \mu \mathrm{m}$ & $20 \mu \mathrm{m}$ \\
Low_mic_b & $3 \mu \mathrm{m}$ & $10 \mu \mathrm{m}$ \\
\hline
\end{tabular}

Table III. Comparison between the average values of the hit numbers in three distinct cases: theoretical computation (see $\$ 5.2$ ), G4 simulation without cuts, G4 simulation with cuts. This computation was done for the high stressed $\mathrm{PhC}$ without shield and secondary events and for the pixel volume.

\begin{tabular}{lccc}
\hline Volume & Th. Hit & $\begin{array}{c}\text { Default Cuts } \\
\left\langle n_{\text {impacts }}\right\rangle\end{array}$ & $\begin{array}{c}\text { Chosen Cuts } \\
\left\langle n_{\text {impacts }}\right\rangle\end{array}$ \\
\hline Pixel & 56.43 & $50.45 \pm 10.34$ & $58.18 \pm 12.81$ \\
\hline
\end{tabular}

\subsection{Theoretical COMputation of GCR Flux}

In order to disentangle the contributions from the different simulated parts of the instrument (detector and shield) we evaluated the impact of the GCR flux onto the 'naked' detector (without spacecraft shielding). In Table III we report the results from the simulated GCR flux hitting the detector only.

We computed the number of impacts, $n_{\text {impacts }}$, as $n_{\text {impacts }}=\pi \Phi S$, where $\Phi$ is the incoming particle flux in $\mathrm{cm}^{-2} \mathrm{~s}^{-1} \mathrm{sr}^{-1}$ ), and $S$ is the total surface area of the volume considered.

The hit average values in the two cases (with G4 default cut values and with our cuts) are compared to those compute theoretically.

Fixing specific cuts increases the error bars, but $\left\langle n_{\text {impacts }}\right\rangle$ comes closer to the expected mean value. The chosen cut values are a fine tuning of the Erg-Al cuts and, as a result, this leads to a very precise tracking inside the detector volumes. 


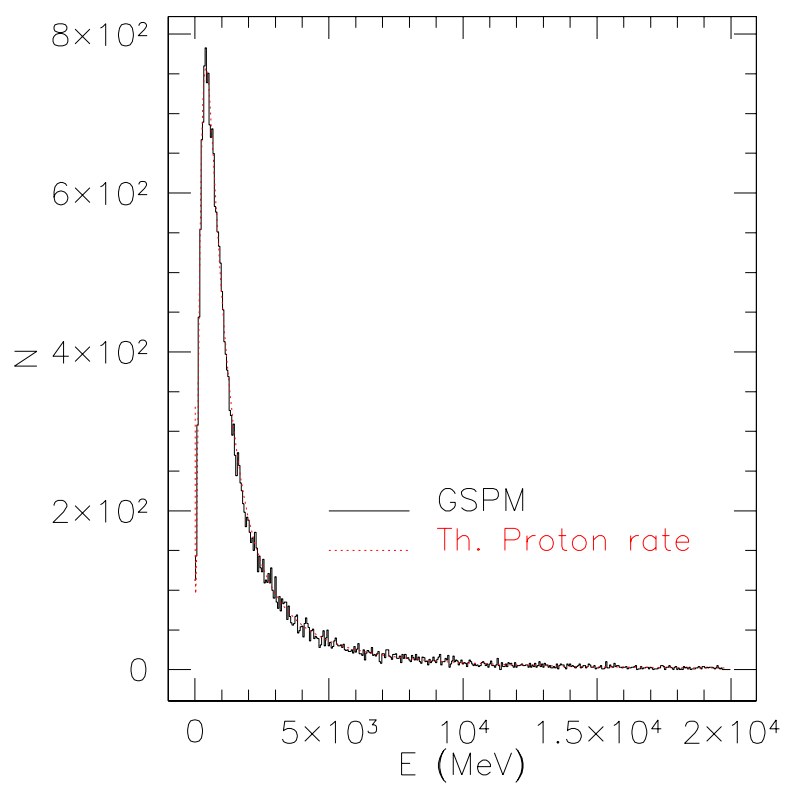

Figure 4. The superposition of theoretical proton rate at solar minimum (dotted line) with the sum of five simulations with GSPM (continuous line). GSPM reproduces very well the GCR spectral energy distribution.

\section{Results from GCR simulations}

\subsection{Preliminary Tests}

To test the correctness of our results we first made some preliminary tests.

The goodness of the internal randomness of GSPM was checked as follows. In Figure 4 both the theoretical (CREME94) and the G4 output of the GSPM energy distributions are shown. Five simulations with $5 \times 10^{3}$ particles each were run and the composite output is compared to the theoretical normalized one (see Figure 3).

\subsection{THE $n_{\text {sec }} / n_{\text {prim }}$ RATIO}

Table IV reports the number of secondary particles produced on average per primary event. The $n_{\text {sec }} / n_{\text {prim }}$ ratio is clearly increasing with the atomic number of the primary particle and the high and low stressed $\mathrm{PhC}$ have rather the same dependence. Unfortunately such a ratio is strictly dependent on the chosen physics list and on the reliability of the G4 models. G4 has been tested against nuclear data for nuclei lighter than or equal to $\mathrm{C}$, therefore the ratios results on $\mathrm{N}$ and $\mathrm{O}$ must be taken with caution. 
Table IV. The $n_{\text {sec }} / n_{\text {prim }}$ ratio from the simulation of GCRs radiation on the photoconductors. In the first column the GCR type; in column \#2 and \#3 the average of secondary events generated by a single primary event for the high stressed $\mathrm{PhC}$ in case of solar min and max irradiation; in column \#4 and \#5 same as \#2 and \#3, but for the low stressed $\mathrm{PhC}$.

\begin{tabular}{lcccc}
\hline & \multicolumn{2}{c}{ High Stressed } & \multicolumn{2}{c}{ Low Stressed } \\
GCR & $\begin{array}{c}\text { Solar min } \\
\left\langle n_{\text {sec }}\right\rangle / n_{\text {prim }}\end{array}$ & $\begin{array}{c}\text { Solar max } \\
\left\langle n_{\text {sec }}\right\rangle / n_{\text {prim }}\end{array}$ & $\begin{array}{c}\text { Solar min } \\
\left\langle n_{\text {sec }}\right\rangle / n_{\text {prim }}\end{array}$ & $\begin{array}{c}\text { Solar max } \\
\left\langle n_{\text {sec }}\right\rangle / n_{\text {prim }}\end{array}$ \\
\hline $\mathrm{H}$ & $3.302 \pm 0.122$ & $4.488 \pm 0.157$ & $3.272 \pm 0.088$ & $4.487 \pm 0.127$ \\
$\mathrm{He}$ & $6.569 \pm 0.265$ & $10.612 \pm 0.279$ & $8.672 \pm 0.241$ & $10.385 \pm 0.335$ \\
$\mathrm{Li}$ & $14.665 \pm 0.744$ & $17.409 \pm 0.416$ & $14.833 \pm 0.269$ & $17.394 \pm 0.493$ \\
$\mathrm{C}$ & $35.859 \pm 0.818$ & $45.489 \pm 0.766$ & $36.473 \pm 0.913$ & $45.844 \pm 1.105$ \\
$\mathrm{~N}$ & $45.527 \pm 1.819$ & $57.420 \pm 0.913$ & $46.275 \pm 1.212$ & $57.658 \pm 1.015$ \\
$\mathrm{O}$ & $55.863 \pm 1.394$ & $70.275 \pm 1.188$ & $55.379 \pm 1.745$ & $72.563 \pm 1.234$ \\
\hline
\end{tabular}

\subsection{Deposited Energy, Definition of A GLitch}

A glitch on the photoconductor is an unexpected voltage jump during the integration ramp. Along a single ramp, that lasts $300 \mathrm{~ms}$, the voltage with a 64 readout sequences decreases monotonically. When the voltage jump exceeds by $4-6$ times (in sigma units) the mean jump we have a glitch.

The exact electronic behaviour is not reproducible with Geant4, we only collected the energy depositions on the pixels. The energy deposited on the pixels is transformed (Groenewegen 2004) in detector signal (Volt):

$$
\Delta E(M e V)=C \Delta V(V o l t) E_{g} /(R h \nu / \eta)
$$

where the photoconductive gain $\mathrm{R}=30 \mathrm{~A} / \mathrm{W}$, at $\lambda=170 \mu \mathrm{m}(\eta=0.3$, with a large error bar); $\mathrm{E}_{g}$ is the energy gap, and it is equal to $2.9 \mathrm{eV}$, and $\mathrm{C}$ is the detector capacity. For $\mathrm{C}=3 \mathrm{pF}$, we get: $\Delta V=0.0134 \Delta E$.

Impacts on pixels are identified and used to compute the deposited energy. Tables ${ }^{5} \mathrm{~V}$ and VI report the mean deposited energy (in V) for both (high and low) stressed $\mathrm{PhC}$ for shielded simulations, i.e. those simulating the effect of the spacecraft.

\footnotetext{
5 Tables report the sum of the voltage jumps normalized to a second of GCR impacting. This value must be corrected by the readout and ramp lasting.
} 
Table V. Mean number of hits and energy deposition expressed in $\mathrm{MeV}$, for the high stressed pixels with spacecraft shielding, secondary events at solar minimum and maximum.

\begin{tabular}{lcccc}
\hline \multicolumn{5}{c}{ High Stressed pixel } \\
GCR & $\langle n\rangle$ & $\left\langle d E_{\text {dep }}\right\rangle(\mathrm{MeV})$ & $\langle n\rangle$ & $\begin{array}{c}\text { Solar max } \\
\end{array}$ \\
\hline $\mathrm{H}$ & $101.41 \pm 5.68$ & $60.21 \pm 3.19$ & $50.86 \pm 0.84$ & $27.35 \pm 1.83$ \\
$\mathrm{He}$ & $17.46 \pm 0.66$ & $23.72 \pm 0.88$ & $8.77 \pm 0.23$ & $9.31 \pm 0.09$ \\
$\mathrm{Li}$ & $0.019 \pm 0.001$ & $0.32 \pm 0.01$ & $0.089 \pm 0.004$ & $0.12 \pm 0.01$ \\
$\mathrm{C}$ & $2.24 \pm 0.07$ & $4.64 \pm 0.18$ & $1.15 \pm 0.04$ & $1.86 \pm 0.09$ \\
$\mathrm{~N}$ & $0.76 \pm 0.02$ & $1.54 \pm 0.06$ & $0.39 \pm 0.01$ & $0.64 \pm 0.01$ \\
$\mathrm{O}$ & $3.23 \pm 0.09$ & $7.07 \pm 0.27$ & $1.74 \pm 0.04$ & $2.89 \pm 0.15$ \\
\hline
\end{tabular}

Table VI. Same as Table V for the low stressed pixels.

\begin{tabular}{lcccc}
\hline & \multicolumn{4}{c}{ Low Stressed pixel } \\
GCR & $\langle n\rangle$ & $\left\langle d E_{\text {dep }}\right\rangle(\mathrm{MeV})$ & $\langle n\rangle$ & $\begin{array}{c}\text { Solar max } \\
\left\langle d E_{\text {dep }}\right\rangle(\mathrm{MeV})\end{array}$ \\
\hline $\mathrm{H}$ & $105.29 \pm 5.63$ & $61.74 \pm 4.42$ & $47.98 \pm 0.95$ & $24.80 \pm 1.54$ \\
$\mathrm{He}$ & $17.59 \pm 0.49$ & $24.21 \pm 0.99$ & $8.66 \pm 0.37$ & $9.41 \pm 0.59$ \\
$\mathrm{Li}$ & $0.019 \pm 0.003$ & $0.332 \pm 0.001$ & $0.087 \pm 0.001$ & $0.119 \pm 0.003$ \\
$\mathrm{C}$ & $2.22 \pm 0.05$ & $4.62 \pm 0.05$ & $1.18 \pm 0.03$ & $1.90 \pm 0.08$ \\
$\mathrm{~N}$ & $0.78 \pm 0.01$ & $1.63 \pm 0.09$ & $0.399 \pm 0.001$ & $0.64 \pm 0.03$ \\
$\mathrm{O}$ & $3.33 \pm 0.05$ & $7.36 \pm 0.21$ & $1.72 \pm 0.04$ & $2.85 \pm 0.19$ \\
\hline
\end{tabular}

\subsubsection{High stressed Photoconductor}

We considered first the effects on the high stressed $\mathrm{PhC}$ of the primary particle impacts on the shield and the detector, and the production of secondary events from the shield and the detector. We ran five simulations for each nucleus. Each simulation considers $2 \times 10^{5}$ particles. Results (see Table V) have been normalized to $1 \mathrm{~s}$ of irradiation.

\subsubsection{Low Stressed Photoconductor}

We ran five simulations for each nucleus. Each simulation considers $2 \times 10^{5}$ particles. Results (see Table VI) have been normalized to $1 \mathrm{~s}$ of irradiation. 
Table VII. First column: number of pixel affected by the negative glitch. Other columns: the mean percentage ( 5 simulations) of GCRs affecting a fixed pixel length, in the shield+secondary events configuration, for each GCRs type, and for the high stressed PhC. Similar values are found for low stressed PhC, and at solar maximum.

\begin{tabular}{ccccccc}
\hline Length & \multicolumn{5}{c}{$\langle\mathrm{G} 4 \%\rangle$} \\
& $\mathrm{p}$ & $\alpha$ & $\mathrm{Li}$ & $\mathrm{C}$ & $\mathrm{N}$ & $\mathrm{O}$ \\
\hline 2 & $3.8_{-2.7}^{+2.7}$ & $5.4_{-1.6}^{+1.6}$ & $5.6_{-2.2}^{+2.2}$ & $7.5_{-2.6}^{+2.6}$ & $6.2_{-1.4}^{+1.4}$ & $7.6_{-1.8}^{+1.8}$ \\
3 & $0.6_{-0.6}^{+1.2}$ & $0.9_{-0.9}^{+2.1}$ & $0.7_{-0.7}^{+0.9}$ & $1.0_{-1.0}^{+1.3}$ & $1.8_{-1.1}^{+1.1}$ & $1.0_{-0.8}^{+0.8}$ \\
4 & - & - & - & $0.3_{-0.3}^{+0.5}$ & $0.1_{-0.1}^{+0.3}$ & $0.1_{-0.1}^{+0.3}$ \\
\hline
\end{tabular}

\subsection{Cross talks}

We checked whether in the G4 outputs there are coincidences (cross talks) between adjacent pixels. The number of pixels affected by one single GCR as resulting from G4 simulations are listed in Table VII for the high stressed $\mathrm{PhC}^{6}$. Values are given in percentage for each GCR type producing cross talks. The percent probability that nearby pixels are affected by a hit is lower than the values given in Table VII.

\subsection{SECONDARY Events}

The formation of secondary events depends strictly on the physics we considered. In case of proton GCRs we obtained a lot of secondary events, from photons and electrons to a large sample of particles (i.e. $\pi^{+}$) to some nuclei (i.e. ${ }^{27} \mathrm{Al}$ ). For the light nuclei, secondary events are basically photons or electrons. Ion inelastic scattering may also activate and generate pions, protons and electrons and smaller nuclei, but these events are much rarer.

Figure 5 reports the number of impacts on the detector in a shielded and unshielded configuration (high stressed $\mathrm{PhC}$ ). The shield does not act as the major source of secondary events and does not largely affect the pixels. Ge pixels are well embedded within other detector components masking the shield, the origin of the secondary.

As expected, the $n_{\text {sec }} / n_{\text {prim }}$ ratio is high because of the large mass of the detector. The ratio is almost identical for high and low stressed $\mathrm{PhC}$, since the two arrays are very similar.

\footnotetext{
${ }^{6}$ No significant dependence on solar activity or on stress status is found.
} 


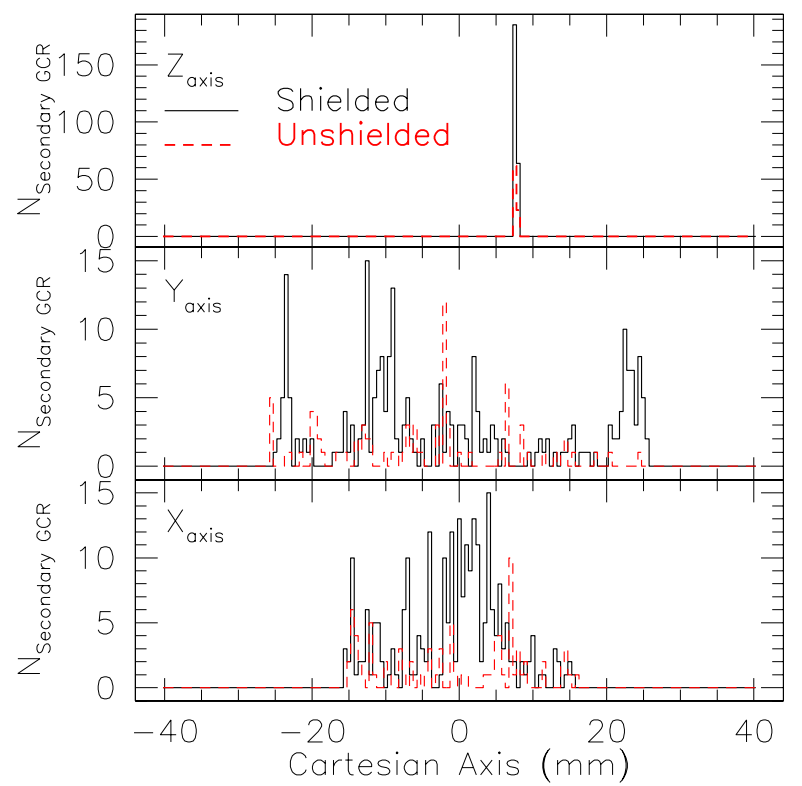

Figure 5. Cartesian projection of secondary events generation space for the high stressed PhC (from 5000 protons seed) for shielded (straight line) and unshielded (dashed line) configurations. Upper panel; the projection along the $\mathrm{Z}$ axis, mid and lower panels projection along the $\mathrm{Y}$ and $\mathrm{X}$-axis.

\section{Proton irradiation tests}

At the Light Ion Facility of the Centre de Recherches du Cyclotron of the University Catholique de Louvain La Neuve, Belgium, some tests were performed, intended to determine the glitch event rate on a single photoconductor module. The 2004 tests could not be reproduced because of sudden unexpected responsivity changes which made impossible to follow the detector behaviour. In addition the external environment was not clearly defined and simulations could not usefully compared to measurements. Tests were repeated in April 2005 and the responsivity jumps were cured by heating the detectors (see Katterloher et al. 2005). Here we report on these latter tests.

\subsection{Test Specimen}

The test specimen foreseen for the proposed investigations under proton irradiation is the detector module FM\#12 with a CRE of the Qualification Model type, mounted in the centre of the module. The detector is in the high stress configuration. The module stood in a liquid He dewar operating at a temperature of $(1.85 \pm 0.05) \mathrm{K}$.

The dewar is made of three concentric cylinders of $\mathrm{Al}$ (called hereafter 


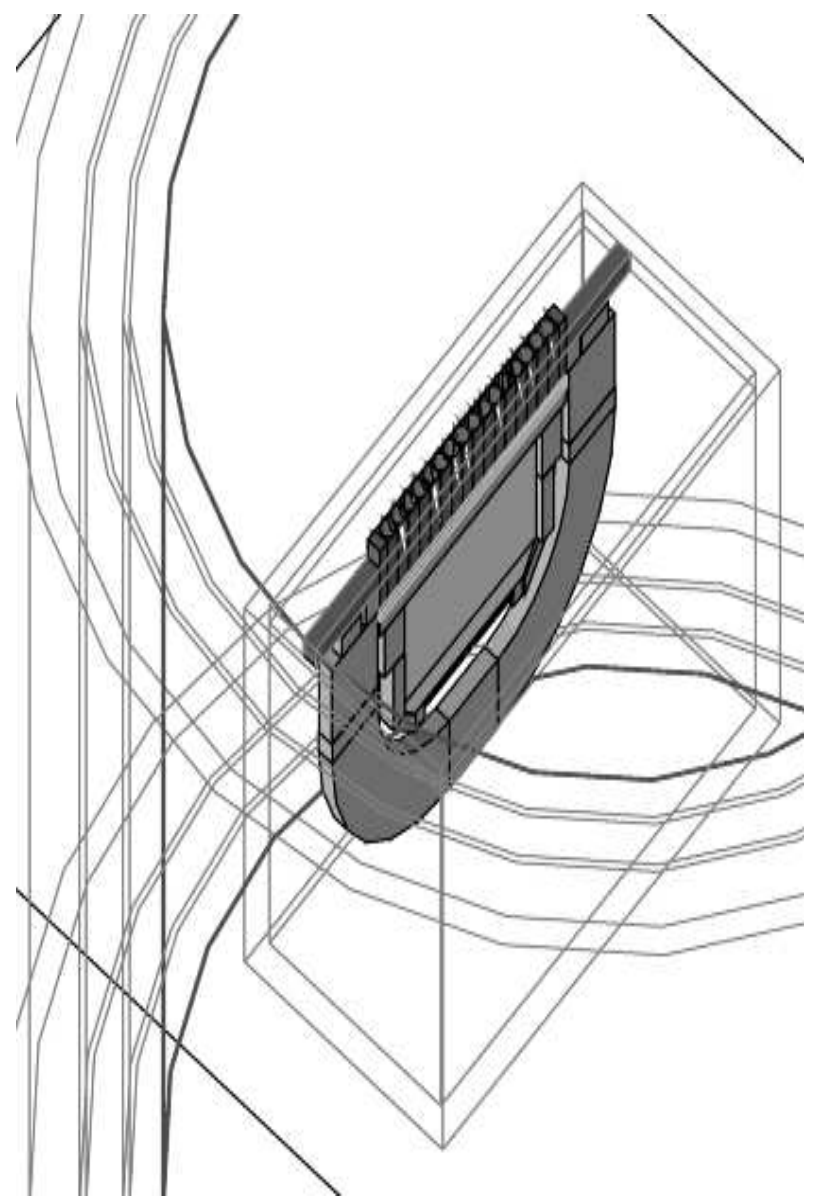

Figure 6. The dewar, and a single stressed module, in the Louvain cyclotron configuration. In gray the three $\mathrm{Al}$ cylinders and the box around the module, in red the $\mathrm{Cu}$ cylinder foil.

$\mathrm{Al1}, \mathrm{Al} 2$ and $\mathrm{Al} 3$ ) and one made of $\mathrm{Cu}$. Al1 is $6 \mathrm{~mm}$ thick. Al2 and $\mathrm{Al} 3$ are $1.5 \mathrm{~mm}$ thick. The $\mathrm{Cu}$ cylinder is $0.5 \mathrm{~mm}$ thick. The module is placed inside a box of $\mathrm{Al} 4 \mathrm{~mm}$ thick ( $\mathrm{Al}$ case) so that half the foreoptics stands outside of it (see Fig. 6). The module is not placed at the centre of the cylinders.

The proton beam, before reaching the Ge:Ga crystals elements, penetrates two layers, one made of steel, $60 \mu \mathrm{m}$ thick, and one made of lead $0.12 \mathrm{~mm}$ thick. There is also a couple of steel collimators, that we modeled as a box, with a hole in its centre as large as the beam.

During the test a primary circular $(10 \mathrm{~cm} \emptyset)$ proton beam of $70 \mathrm{MeV}$ was used, with a beam length of $5.12 \mathrm{~m}$ between the diffusion foil and the Device Under Test (DUT). The beam reaches the dewar (and the 


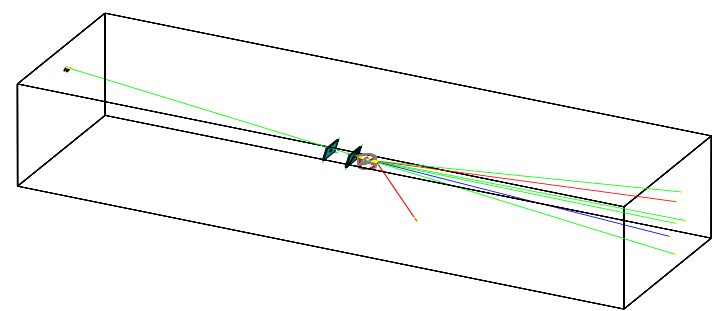

Figure 7. The UCL-CRC experimental hall. The detail of the DUT (here in the center of the plot) is in Figure 6.

module) under an angle of $10^{\circ}$ (see Figure 7 ). The DUT is placed into air. Due to air leakage, we set air cuts as large as we can, in order not to have a massive secondary event generation, that is $2.5 \mathrm{~m}$.

\subsection{The G4 Simulations}

We first ran 5 simulations of 10000 protons each, in order to have an idea of what occurs to the beam once it crosses all the layers. We assume that the beam had a Gaussian error of $1 \mathrm{MeV}$. Before the first $\mathrm{Al}$ cylinder layer we had an energy of $\approx 58.09 \mathrm{MeV}$, which disagrees with data by J. Cabrera, $63.74 \mathrm{MeV}$ (Katterloher et al. 2005). This is 
Table VIII. Summary of proton seed and photo-conductor module setup during UCL-CRC experiments. Column \# 1 reports the identifier of the chosen setup, \# 2 the value of the bias voltage of the FEE circuit, \# 3 the duration of each integration ramp, \# 4 the integrator capacitor, \# 5 the number of ramps, \# 6 the number of repetition with the same measurement setup, \# 7 the proton flux.

\begin{tabular}{lrrrrrr}
\hline Exp. & $\begin{array}{r}\text { BIAS } \\
(\mathrm{mV})\end{array}$ & $\begin{array}{r}\mathrm{t}_{\text {ramps }} \\
(\mathrm{ms})\end{array}$ & $\begin{array}{r}\mathrm{C} \\
(\mathrm{pF})\end{array}$ & $\mathrm{N}_{\text {ramps }}$ & $\mathrm{N}_{\text {rep }}$ & $\begin{array}{r}\text { Flux } \\
\left(\mathrm{cm}^{-2} \mathrm{~s}^{-1}\right)\end{array}$ \\
\hline \#L1 & 50 & 250 & 1.09 & 1024 & 35 & 10 \\
& 50 & 250 & 1.09 & 1300 & 3 & 10 \\
& 30 & 250 & 0.23 & 1024 & 23 & 10 \\
& 20 & 250 & 0.23 & 1024 & 4 & 10 \\
& 40 & 250 & 0.23 & 1024 & 1 & 10 \\
& 50 & 250 & 0.23 & 1024 & 1 & 10 \\
& 70 & 250 & 0.43 & 1024 & 1 & 10 \\
& 30 & 250 & 0.23 & 512 & 2 & 10 \\
\hline \#H1 & 30 & 250 & 0.23 & 1024 & 18 & 400 \\
\hline
\end{tabular}

due to the new G4 version we used $\left(6.2 .02^{7}\right.$, gave $\left.\approx 63.70 \mathrm{MeV}\right)$. Our modelled dewar is reliable and represents closely the dewar geometry. We do not have reasons to believe the Geant4 fails in this case and we set the energy hitting the module at $15.22 \pm 1.95 \mathrm{MeV}$ (bottom right panel of Figure 8). In Figure 8 we plot the degradation of the proton energy along the path inside the dewar.

What we would like to stress here is that 10000 protons originate roughly 22300 secondary events. This is mainly due to the air leakage. After the $\mathrm{Al}$ box, $\approx 610$ protons survive. But, after the $\mathrm{Al}$ box, there are also $\approx 330$ secondary events. The spectral range and type of these secondary events is strictly dependent of the reliability of the G4 models.

In Table VIII we give the relevant information on the UCL-CRC experiment as performed at the Louvain cyclotron (Katterloher et al. 2005).

\footnotetext{
7 Furthermore the 6.7.01 version gave a very rare crash, probably due to a bug in the Hadronic Physics for the High Precision neutrons: with this new version we switched off such a physics.
} 



Figure 8. Degradation of the proton energy across the dewar layers: from the top-left panel, the energy of the incoming proton flux, the flux emerging from the polystyrene foil, after the Aluminum shields, after the $\mathrm{Cu}$ case and the final detector case. The last panel refers to the energy deposition on the Ge:Ga material.

\subsection{The Geant4 Results}

We ran the same experiments under the G4 tool. Results are summarized in Table IX. In Fig. 9 we plot the energy deposition onto the 16 pixels in the $\mathrm{H} 1$ test.

Here we found 3 peaks. The major one, is clearly due the primary proton beaming. The one at low energies, is due to the secondary events generated along the tracking of the protons along all the components we described before. The peak at higher energy could be due to the fact that the pixel are inside a non uniform cavity: due to the inclination of 


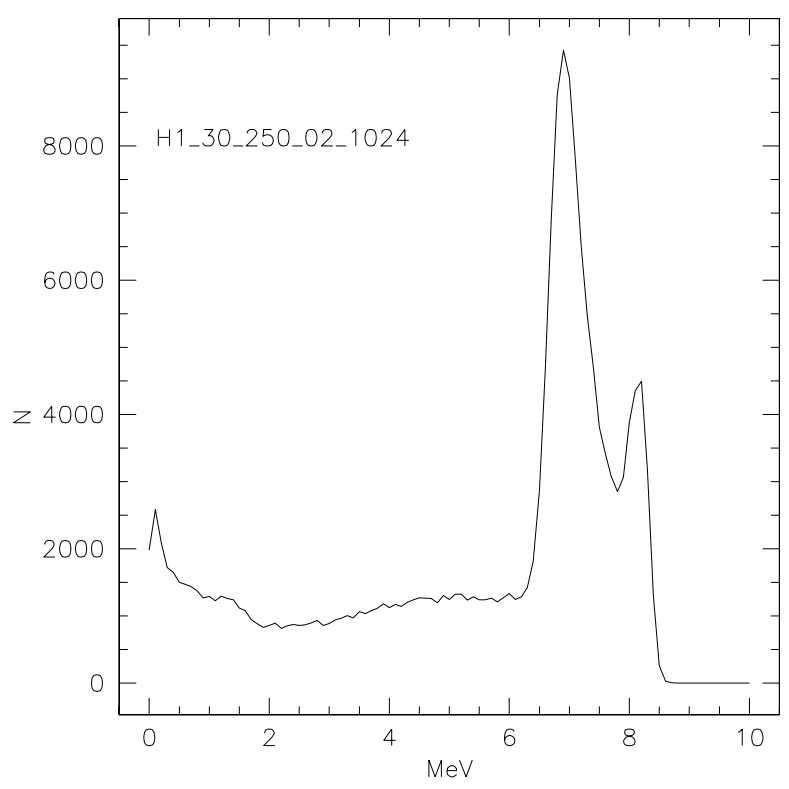

Figure 9. Energy deposition for the H1 test.

Table IX. The UCL-CRC experiment outputs with Geant4.

\begin{tabular}{rrrrrrrr}
\hline Exp. & $\mathrm{N}_{\text {rep }}$ & Geant4 rep & $\begin{array}{r}\text { Flux } \\
\left(\mathrm{cm}^{-2} \mathrm{~s}^{-1}\right)\end{array}$ & $\begin{array}{r}\mathrm{t}_{\text {rad }} \\
(\mathrm{s})\end{array}$ & $\mathrm{N}_{p}$ & $\langle$ events $\rangle$ & $\begin{array}{r}\text { Rate } \\
\left(\mathrm{s}^{-1} \mathrm{~cm}^{-2}\right)\end{array}$ \\
\hline \#L1 & 35 & 35 & 10 & 256 & 200960 & 251.83 & 3.53 \\
& 3 & 6 & 10 & 325 & 255125 & 328.00 & 3.63 \\
& 23 & 23 & 10 & 256 & 200960 & 252.87 & 3.55 \\
& 4 & 10 & 256 & 200960 & 255.38 & 3.58 \\
& 1 & 5 & 10 & 256 & 200960 & 267.20 & 3.75 \\
& 1 & 5 & 10 & 256 & 200960 & 250.60 & 3.52 \\
& 1 & 5 & 10 & 256 & 200960 & 253.20 & 3.55 \\
& 2 & 10 & 10 & 128 & 100480 & 129.10 & 3.62 \\
\hline \# 1 & 18 & 18 & 400 & 256 & 8038400 & 9948.06 & 139.58 \\
\hline
\end{tabular}

the beam there is a part of it that cross the cavity in its thinner part, that is: pixel are hit by more energetic protons.

\subsection{COMPARISON With MEASUREMENTS}

A preliminary analysis of these data was made by Groenewegen \& Royer (2005). Here we report the results in energy; the detector output 
signal in Volts can be retrieved from equation 1. In the report by Groenewegen \& Royer (2005) the conversion value between $\mathrm{MeV}$ and Volt is taken to be 1.34 .

\subsubsection{Events and Rates}

The number of events observed in files L26-L29 were 755, corresponding to a rate of $3.1 \mathrm{~s}^{-1} \mathrm{~cm}^{-2}$. If corrected for the efficiency of the detection algorithm it becomes $3.9 \mathrm{~s}^{-1} \mathrm{~cm}^{-2}$. For such a dataset, G4 finds 853 events and a rate of 3.53. The number of events observed in files H3-H6 is 29008, G4 has 29430 events. Within the uncertainties these values are similar. The fundamental physical processes occurring in this experiment are therefore reproduced.

\subsubsection{Deposited Energy}

In Figures 9 and 10 we plot the distribution of the predicted deposited energy for the files L26, L27, L28 and L29 and those at large proton flux $\mathrm{H} 1$ in MeV. The energy distribution of the glitches, i.e. that of the number of hits reaching the detector module, shows that many glitches occur at low energy and the distribution is asymmetric with a peak at $\approx 7 \mathrm{MeV}$. The distributions are bimodal and have a second peak around $8 \mathrm{MeV}$ (see $\S 7.3$ ) The energy glitch distribution is, as expected, the same in both experiments (H1 and L26-L29) with low proton and high proton fluxes (Figures 9 and 10). What is changing is only the number of hits which in the second case is hundreds times lower. In fact, the proton energy is the same, in both configurations, only the beam intensity is changing.

Figure 11 shows, in the upper panel, the measured glitch distribution in Volt, and in the lower panel the high energy tail of the glitch distribution shown in Figure 10. We apply here the same deglitching algorithm and get rid of the low energy tail of the hits. This figure, i.e. the glitch detection, depends strictly on the algorithm used to deglitch the data (Table 9 of Groenewegen \& Royer 2005). If, for instance, we applied a cutoff value around $\approx 6 \mathrm{MeV}$ the distribution of glitches would be more similar to the observed one. This latter has a peak at low energy, with a rather long tail towards high energies (Groenewegen \& Royer 2005). The simulated distribution gave first the tail, then the two peaks and is much narrower (cfr. $\S 7.3$ ).

To compare the two distributions a conversion factor to the $\mathrm{x}$ axis values (either to $\mathrm{MeV}$ or to Volt) must be applied. This can be accomplished using eq. (10). The resulting value is, in our case, $\Delta E(M e V)=6.71 \Delta V(V)$. This value was computed from the measurements taking into account the responsivity changes under irradiating conditions (Lothar Barl, private communication). The two distributions 




Figure 10. The energy distribution of glitches for files L26-L29, with Geant4.

however differ substantially, the simulated one being much narrower. The difference could be due to a large number of secondary events produced during the tests by some unknown components we are not aware of. This component should have a large energy and it is unlikely that it occurs but this hypothesis cannot be discarded. Another possible explanation is that the conversion factor from $\mathrm{MeV}$ to Volt is a function of the energy. Although plausible, at present we cannot prove this possibility.

\subsubsection{Boundary Effect}

Figure 12 shows the number of detected glitches per detector: a clear boundary effect (i.e. external pixels of the module were under-hit with respect to the central ones) is seen, and is due to a differential incident proton flux with respect to the detector position (Figure 12 in Katterloher et al. 2005). We find a difference of the hit numbers of $20 \%$ while the measured beam intensity difference between the central pixel and the outer ones is $10 \%$. This is a geometrical effect and does not correspond to a different behaviour of the single chip. 

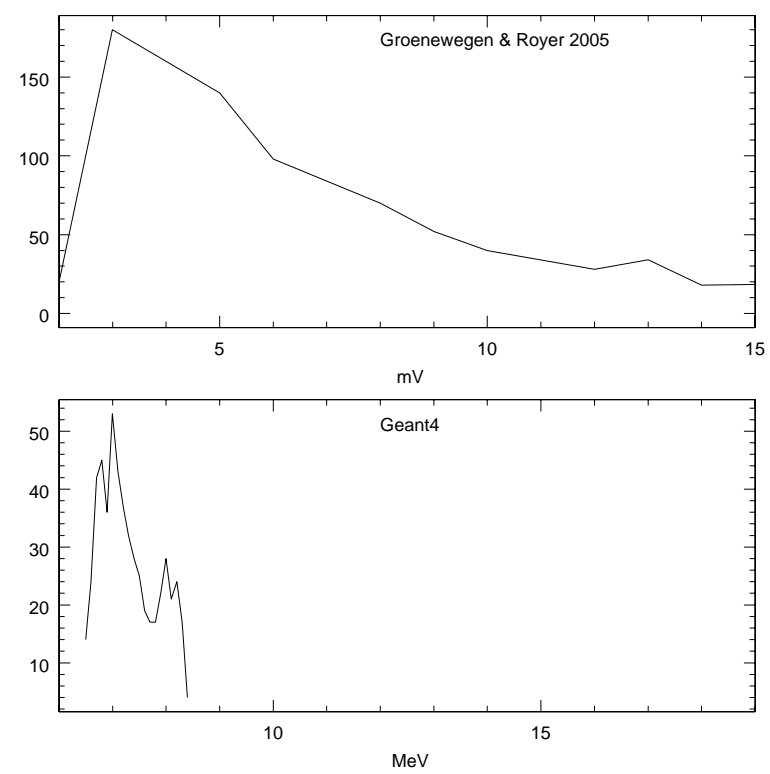

Figure 11. Upper panel: the observed distribution of glitches once a deglitching algorithm has been applied. Lower panel: the G4 output of the same measuring setup and the same deglitching algorithm.

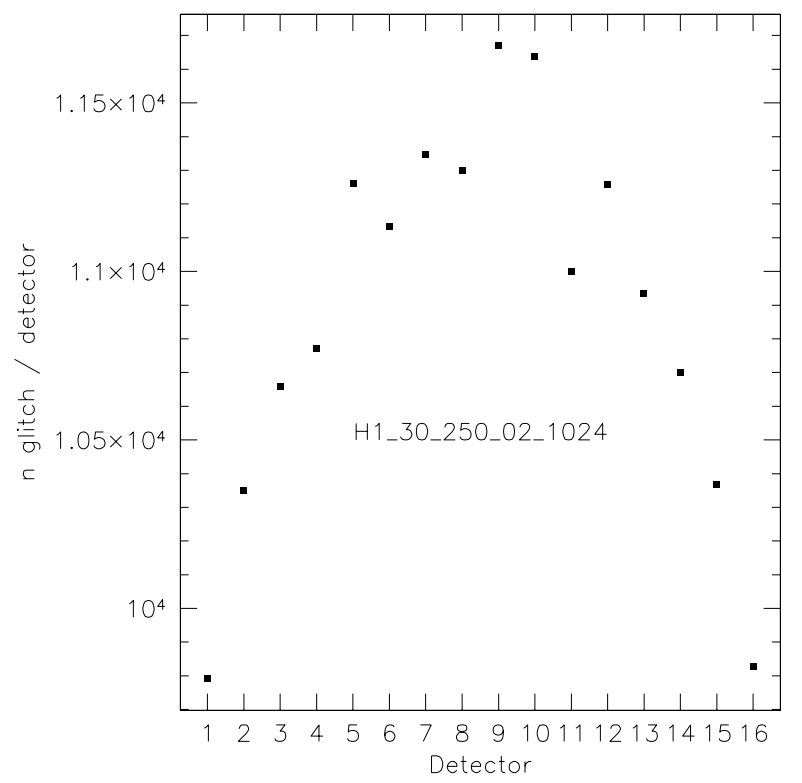

Figure 12. The boundary effect. Number of G4 simulated glitches per detector (pixel) in the $\mathrm{H} 1$ setup. 


\section{Discussion}

\subsection{The PHotoconduCtors In the SPACE ENVIRONMENT}

Our simulations of the effects of Galactic Cosmic Ray impacts on the photoconductor arrays show that both the satellite plus the cryostat (the shield) and the detector act as source of secondary events, affecting the detector performance. Secondary event rates originated within the detector and from the shield are of comparable intensity. The impacts deposit energy on each photoconductor pixel but do not affect the behaviour of nearby pixels. These latter are hit with a probability always lower than $7 \%$.

The energy deposited produces a spike which can be hundreds times larger than the noise. The present simulations are not able to follow the temporal behaviour of each pixel and cannot be used to determine the shape of the output signal as a function of time.

\subsection{The PHOTOCONDUCTORS IN THE LAB TEST}

We have simulated the experiment carried out at UCL-CRC and compared the simulation outputs to the measurements reported in Groenewegen \& Royer (2005). We find similar rates and events (see Tables $\mathrm{X}$ ) on each pixel. The simulated energy distribution differs substantially from that of the measurements.

We tried to ascribe these differences to the following causes:

1. As far as the event number and rate is concerned, it may be possible that the chosen cuts are rather large. But due to the complexity of the experimental hall it is hard to tune them optimally. We would need information on the intermediate passage of the beam through matter. A simple correction could be a slight increase of the Ge cuts. A clear benchmark would be the comparison between the energy distributions.

2. The presence of a primary and a secondary peak seems to be the only feature common between experimental and simulated data. What it is not clear is why, due to the crossing through matter, the Gaussian beam is (should be) transformed as in Figure 8, that is first a tail, then a peak, whereas experimental data favour the tail next to the secondary peak. Either Ge has some physical properties which are not included in the G4 Physics List, or the deglitching algorithm used is not correct. 
Table X. Measured versus predicted values.

\begin{tabular}{rrr}
\hline Files & $\begin{array}{r}\text { (Groenewegen \& Royer 2005) } \\
\text { Events }\end{array}$ & $\begin{array}{r}\text { Geant4 } \\
\text { Events }\end{array}$ \\
\hline L26-L29 & 755 & 853 \\
H3-H6 & 29008 & 29430 \\
\hline
\end{tabular}

\section{Acknowledgements}

We warmly thank M. Asai, G. Cosmo, A. De Angelis, F. Lei, V. Ivantchenko, G. Santin and J.P. Wellish, who helped us in this effort and elucidating us the tricks of the Geant4 code. We thank Lothar Barl for his useful information about the photoconductor detector.

\section{References}

Bertini H.W., Githrie P. 1979, Nuclear Physics, 169 Results from Medium-Energy Intra-Nuclear-Cascade Calculation

Ferguson C., 2000a, Uos-GSPM-SSD, General Purpose Source Particle Module for GEANT4/SPARSET: Software Specification Document web-page:

reat.space.qinetiq.com/gps/gspm_docs/gspm_ssd.pdf

Ferguson C., 2000b, Uos-GSPM-Tech, General Purpose Source Particle Module for GEANT4/SPARSET: Technical Note web-page:

reat.space.qinetiq.com/gps/gspm_docs/gspm_tn1.pdf

Ferguson C., 2000c, Uos-rep-07, General Purpose Source Particle Module for GEANT4/SPARSET: User Requirement Document, web-page:

reat.space.qinetiq.com/gps/gspm_docs/gspm_urd.pdf

Groenewegen M., 2004, PICC-KL-TN-012 UCL-CRC Proton Tests of March 2004: glitch height distribution

Groenewegen M. \& Royer P., 2005, PICC-KL-TN-020 Analysis of the April 2005 proton test data

Katterloher R., Barl L., \& Shubert J., Test Plan and Procedures for Investigation of Glitch Event Rate and Collected Charge Variation in the Ge:Ga Detectors during Proton Irradiation at UCL-CRC (2 ${ }^{\text {nd }}$ test phase, PACS-ME-TP-009, 2004

Ivanchenko V., Folger G., Wellish J.P., Koi T., Wright D.H., 2003, Computing in High Energy and Nuclear Physics, La Jolla, California, SLAC-PUB-9862

Nymmik R.A., Panasyuk M.I., Pervaja T.I., \& Suslov A.A., 1992, Nuclear Tracks and Radiation Measurements, 20, 427

Prantzos N. Li, Be, B and Cosmic Rays in the Galaxy, astro-ph/0411569

Shen W.-Q., Wang B., Feng J., Zhan W.-L., Zhu Y.-T., \& Feng E.-P.,1989, Nuc. Phys. A, 491, 130

Tripathi, R.K., Wilson, J.W. and Cucinotta, F.A. 2000, Nasa Technical Paper 3621 
gegapaper2_rev.tex; 6/12/2018; 20:50; p.26 